\title{
Neurocognitive deficits related to poor decision making in people behind bars
}

\author{
ELDAD YECHIAM \\ Technion-Israel Institute of Technology, Haifa, Israel \\ Jason E. KANZ AND ANTOINE BEChARA \\ University of Iowa, Iowa City, Iowa \\ Julie C. Stout And Jerome R. Busemeyer \\ Indiana University, Bloomington, Indiana \\ AND \\ Elizabeth M. Altmaier and Jane S. Paulsen \\ University of Iowa, Iowa City, Iowa
}

\begin{abstract}
Using a novel quantitative model of repeated choice behavior, we investigated the cognitive processes of criminal offenders incarcerated for various crimes. Eighty-one criminals, including violent offenders, drug and sex offenders, drivers operating a vehicle while impaired, and 18 matched controls were tested. The results were also contrasted with those obtained from neurological patients with focal brain lesions in the orbitofrontal cortex and from drug abusers. Participants performed the computerized version of the Iowa gambling task (Bechara, Damasio, Damasio, \& Anderson, 1994), and the results were decomposed into specific component processes, using the expectancy valence model (Busemeyer \& Stout, 2002). The findings indicated that whereas all the criminal groups tended to select disadvantageously, the cognitive profiles exhibited by different groups were considerably different. Certain subpopulations - most significantly, drug and sex offenders - overweighted potential gains as compared with losses, similar to chronic cocaine abusers. In contrast, assault/murder criminals tended to make less consistent choices and to focus on immediate outcomes and, in these respects, were more similar to patients with orbitofrontal damage. The present cognitive model provides a novel way for building a bridge between cognitive neuroscience and complex human behaviors.
\end{abstract}

Do different groups of criminals suffer from the same dysfunctional cognitive processes when making decisions? The present study explores the nature of the cognitive processes of criminal offenders, including violent offenders, drug and sex offenders, and dangerous drivers (operating a vehicle while impaired [OWI]). The study also contrasts the pattern of these cognitive processes with those of neurological patients with bilateral orbitofrontal cortex damage (i.e., ventromedial prefrontal cortex damage), as well as with those of chronic cocaine abusers.

The issue of similarities and differences between criminal populations prevails in the criminology and psychopathy literature. The famous early criminologist Cesare Lombroso (1911/2005) argued that all criminals share the same characteristics and developed the concept of the atavistic, or born, criminal. Although subsequent facts did not fit Lombroso's theory and his research was later questioned, the idea that all criminals have shared characteristics lingers to date. Indeed, one of the more popular theories in criminal research is the self-control theory of Gottfredson and Hirschi (1990), which posits that "people who lack self-control will tend to be impulsive, insensitive, physical (as opposed to mental), risk-taking, short-sighted, and non-verbal" (p. 90). According to Gottfredson and Hirschi's theory, poor self-control is also the primary underlying factor in criminality. Research has indeed shown that poor self-control is associated with a variety of imprudent behaviors, including drug and alcohol use, drunk driving, gang membership, and the use of violence (see, e.g., Armstrong, 2005; Hope \& Damphouse, 2002; Keane, Maxim, \& Teevan, 1993).

The present study espouses a different view, that whereas poor decision making is characteristic of criminal behavior, the cognitive processes that lead to this behavior might vary across criminals who commit different offenses. The idea that grouping criminals according to types of offenses could yield insight into important individual differences in psychological characteristics is not new. For example, several studies in which self-report measures have been used have indicated that different criminal populations appear

J. S. Paulsen, jane-paulsen@uiowa.edu 
to belong to different personality groups (see Krueger, Hicks, \& McGue, 2001). In our study, we asked whether criminals with different primary offenses might have characteristic differences in basic psychological processes that underlie decision making. Building on previous studies in clinical populations using the Iowa gambling task (IGT; Bechara, Damasio, Damasio, \& Anderson, 1994), we and others have found that whereas a wide variety of clinical populations perform more poorly than their respective control groups on this task, the cognitive processes that lead to these poor performances can differ between clinical populations and, thereby, add insight regarding the different pathways to poor decision making that might characterize various disorders.

The utility of this approach has been demonstrated previously (Busemeyer \& Stout, 2002; Yechiam, Busemeyer, Stout, \& Bechara, 2005). The IGT is a laboratory decision-making task in which participants make a series of 100 choices from four decks of cards with the goal of maximizing their accumulated payoff. The decision makers do not know in advance the outcomes associated with each deck. Each card selection from one particular deck leads to monetary gains, but also to intermittent losses (see the Method section and Table 1). Two of the decks are disadvantageous, in that they yield relatively higher gains but incur even larger losses, so that they lead to a net loss. The other two decks are advantageous, since they yield relatively lower gains but also much smaller losses, leading to a net profit.

Prior studies have shown that poor behavioral decisions on the IGT, measured as preference for the disadvantageous decks, could be associated with different psychological components (Busemeyer \& Stout, 2002; Yechiam et al., 2005). For example, one such component is the

Table 1

Schedule of Rewards and Penalties for the Four Decks of Cards: First Block of 10 Selections From Each of the Decks (Out of Six Blocks)

\begin{tabular}{|c|c|c|c|c|}
\hline Card & $\begin{array}{c}\text { Deck A } \\
\text { (Win } \$ 100 \\
\text { Every } \\
\text { Trial) }\end{array}$ & $\begin{array}{c}\text { Deck B } \\
\text { (Win } \$ 100 \\
\text { Every } \\
\text { Trial) }\end{array}$ & $\begin{array}{c}\text { Deck C } \\
\text { (Win } \$ 50 \\
\text { Every } \\
\text { Trial) }\end{array}$ & $\begin{array}{c}\text { Deck D } \\
\text { (Win } \$ 50 \\
\text { Every } \\
\text { Trial) }\end{array}$ \\
\hline 1 & & & & \\
\hline 2 & & & & \\
\hline 3 & $-\$ 150$ & & $-\$ 50$ & \\
\hline 4 & & & & \\
\hline 5 & $-\$ 300$ & & $-\$ 50$ & \\
\hline 6 & & & & \\
\hline $\begin{array}{l}7 \\
8\end{array}$ & $-\$ 200$ & & $-\$ 50$ & \\
\hline $\begin{array}{l}8 \\
9\end{array}$ & $-\$ 250$ & $-\$ 1,250$ & $-\$ 50$ & \\
\hline 10 & $-\$ 350$ & & $-\$ 50$ & $-\$ 250$ \\
\hline Average loss & $-\$ 125$ & $-\$ 125$ & $-\$ 25$ & $-\$ 25$ \\
\hline Frequency of loss & 0.5 & 0.1 & 0.5 & 0.1 \\
\hline Average gain & $\$ 100$ & $\$ 100$ & $\$ 50$ & $\$ 50$ \\
\hline Average gain - loss & $-\$ 25$ & $-\$ 25$ & $\$ 25$ & $\$ 25$ \\
\hline
\end{tabular}

Note-The average gains and losses are across all 10 selections. The gains on each trial range from $\$ 80$ to $\$ 120$ for Decks A and B (normally distributed in discrete steps of $\$ 10$ ) and from $\$ 40$ to $\$ 60$ for Decks C and $\mathrm{D}$ (normally distributed in discrete steps of $\$ 5$ ). tendency to attend to gains and to pay less attention to losses. High weighting of gains, as compared with losses, increases the attractiveness of the disadvantageous decks, since these produce large gains but also large losses. A second relevant component is the tendency to focus on recent outcomes and ignore or rapidly discount past outcomes. Extremely high weighting of recent outcomes can also increase the attractiveness of disadvantageous decks because the infrequent past negative payoffs produced by these decks are discounted. Yet a third component that can lead to poor performance is low choice consistency (or randomness of the choices). These components can be measured using a cognitive model, the expectancy valence (EV) model (Busemeyer \& Stout, 2002), which is a learning model applied to predict the next choice on each trial. The model has three parameters, each corresponding to one of the psychological processes described above: attention to gains versus losses, weighting of recent versus past outcomes, and the degree of choice consistency (deterministic vs. erratic responses). The model is described fully in the Appendix. Using this cognitive model, recent research has shown that although different clinical populations may similarly select disadvantageous decks in the IGT, distinctive clinical deficits are often associated with a diagnostic pattern in the three psychological components of the EV model (see Yechiam et al., 2005).

Using a similar approach, the present study explores the possibility that impairments in distinct psychological processes may explain a common pattern of disadvantageous choices among different types of criminals. Eighty-one prisoners were recruited from the Iowa Medical and Classification Center, including 22 convicted of theft, 4 convicted of OWI, 17 convicted of sex crimes, 22 convicted of drug crimes, 6 convicted of robbery, and 10 convicted of assault/murder. The task performance of each group was compared with that of a sample of matched healthy controls.

In addition, the present study includes previously reported data on patients with brain damage. Theories of impulsive aggression implicate frontal (and especially orbitofrontal) areas in impulsive aggression (Raine, 2002; Raine, Lencz, Bihrle, LaCasse, \& Colletti, 2000). We therefore considered it important to compare the cognitive processes of the criminal samples in our study with those of patients with bilateral orbitofrontal lesions. Specifically, we compared the prisoner data with those of 21 patients with bilateral damage to the orbitofrontal region (Bechara, Damasio, \& Damasio, 2000). In addition, evidence in neurological studies of criminal behavior is often confounded by other factors, such as substance abuse. Indeed, several studies have shown functional and structural abnormalities in the brains of cocaine abusers (see Volkow et al., 1997) that are similar to those of criminal psychopaths. In order to assess the impact of substance abuse on behavior in the absence of known criminal convictions, we also compared the results with previous findings in 12 chronic (5+ years) cocaine abusers, who abstained from drugs for 36-48 h prior to the experiment (Stout, Busemeyer, Lin, Grant, \& Bonson, 2004). 


\section{METHOD}

\section{Participants}

One hundred forty-four first-time incarcerated offenders $(83 \%$ of them male) were randomly chosen from the weekly institutional classification roster and were approached to participate. A compensation (\$3) for research participation was offered, deposited into the inmates' telephone accounts. Fourteen inmates declined to participate, and 34 were excluded. Individuals were excluded because they lacked the necessary institutional tests, had IQ scores less than 60, or had had a recent brain injury. The final sample included 96 offenders ( 17 women and 79 men). These participants were categorized on the basis of their primary sentence into six groups, including (1) theft crimes $(n=22)$, (2) OWI crimes $(n=4)$, (3) sex crimes $(n=17)$, (4) drug crimes $(n=22),(5)$ robbery crimes $(n=6)$, and (6) assault/ murder $(n=10)$. The average sentence was 6 years for theft and OWI crimes, 10 years for sex and drug crimes, 13 years for violent robberies, and 17 years for assault/murder. Fifteen prisoners who did not belong to these groups or had multiple primary sentences were excluded. The prisoners' average age was 29 years (ranging from 18 to 63), and their average education was 11.5 years. This sample was compared with a control sample of 18 participants $(78 \%$ of them male), with an average age of 29 years and 12.5 years of education (see Table 2). The control group was matched for gender, age, and education. The average IQ score for the control group was 8.6 points higher than the average for the criminal group $[t(97)=$ $2.58, p<.05]$. Hence, we covaried the statistical comparison of the two groups for IQ.

In addition, the sample was compared with previously studied brain-lesioned patients (Bechara et al., 2000; Bechara, Damasio, Damasio, \& Lee, 1999) and cocaine abusers (Stout et al., 2004). Because the orbitofrontal patients were somewhat older than the other groups of interest, these patients were compared with a group of older healthy controls (matched for gender, age, and education), whereas all other groups were compared with a group of younger healthy controls (see the bottom section of Table 2). The two control groups of young and mature adults were not significantly different on the model parameters (see the bottom left side of Figure 3 ). The young control group was not significantly different from the control group used in the original cocaine abuse study (Stout et al., 2004), and using this control group replicates the results in Figure 3.

\section{Measures}

We used the computerized version of the IGT (Bechara et al., 1999). In the IGT (Bechara et al., 1994), participants are presented with four card decks (labeled A, B, C, D) and are told to accumulate as much (real) money as possible by picking cards from the decks. Decks differ with respect to the size and frequency of payoffs produced by each card selection. The average gains and losses in the first 10 selections from each deck are described in Table 1. In addition, in the computerized version of the task, differences between decks increase over time. Specifically, the average positive outcome for Decks A and B is increased by $\$ 10$ on each block of 10 trials, whereas the average loss is increased by $\$ 25$. For the advantageous decks, the average gain is improved by $\$ 5$ every 10 trials, as compared with a $\$ 2.5$ increase in the average loss. The probability of loss with Decks A and C is also increased by 0.1 on each block. This was implemented because, when the original task payoffs are used in a computerized test, healthy control subjects sometimes make more choices from the disadvantageous decks than from advantageous decks (see, e.g., Caroselli, Hiscock, Scheibel, \& Ingram, 2006).

The minimum intertrial interval was set to $0.5 \mathrm{sec}$, and the game included 100 trials. The participants were given written instructions identical to those given in Bechara et al. (1999). Briefly, the participants were told that some decks were worse than others and that they should avoid those decks to win the game. They were not given any information about the expected amounts and the proportions of gains and losses.

\section{Procedure}

The participants were individually administered a demographic checklist, the Beta IQ test (Kellogg \& Morton, 1974), and the IGT. The results were analyzed using the EV model (see the Appendix). The model parameters are fitted to maximize the log likelihood of the data, using one-step-ahead predictions of choices based on the previous outcomes obtained by the participant.

In the present study, the accuracy of the EV model was compared with that of two alternative learning models: a simple Softmax model, which bases its prediction on the experienced expected values (e.g., Daw, O'Doherty, Dayan, Seymour, \& Dolan, 2006), and a Softmax model with separate weights to gains and losses (hereafter called Softmax-gains/losses). These models are described in more detail in the Appendix. In addition, we compared the EV model with a simple statistical model that predicts the next choice solely on the basis of the average choice proportions (see Busemeyer \& Stout, 2002). The statistical test we used for comparing the fit of the models was the Bayesian Information Criterion (BIC; Schwartz, 1978) for $\log$ likelihood differences. The BIC is a model-comparison index, based on Bayesian principles, that penalizes models with additional parameters:

$$
\mathrm{BIC}=2 \cdot \log \text { likelihood difference }-k \cdot \ln (N),
$$

where $k$ equals the difference between models in the number of parameters and $N$ equals the number of observations (100). For example, the EV model has three parameters, whereas the simple Softmax model has only one. Thus, $2 \cdot \ln (100) \approx 9.2$. This constitutes the deduction from the fit of the EV model. Positive values of the BIC statistic indicate that the EV model performs better than an alternative model.

\section{RESULTS}

The first analysis simply verified that the prisoners had, indeed, made poor choices on the IGT (see Figure 1). Although both the prisoners and the healthy controls preferred the disadvantageous decks initially, only the control group eventually learned to strongly prefer the advantageous decks. None of the prisoner groups learned to prefer the advantageous decks by the end of the task. A mixed ANOVA with group as an independent variable,

Table 2

Demographic Details of the Participants: Gender Proportion, Average Age, Years of Education, and Beta IQ

\begin{tabular}{clrrrr}
\hline \multicolumn{1}{c}{ Age } & \multicolumn{1}{c}{ Group } & Gender & Age & Education & Beta IQ \\
\hline Young adults & Criminals (81) & $82 \% \mathrm{M}$ & 29 & 11.5 & 97.0 \\
& Controls: Healthy volunteers (18) & $78 \% \mathrm{M}$ & 29 & 12.5 & 105.6 \\
& Cocaine abusers (12) & $100 \% \mathrm{M}$ & 37 & 13.0 & 93.7 \\
\multirow{3}{*}{ Mature adults } & Orbitofrontal patients (21) & $57 \% \mathrm{M}$ & 53 & 12.0 & 99.9 \\
& Controls: Healthy volunteers (20) & $55 \% \mathrm{M}$ & 53 & 12.2 & 101.3 \\
\hline
\end{tabular}




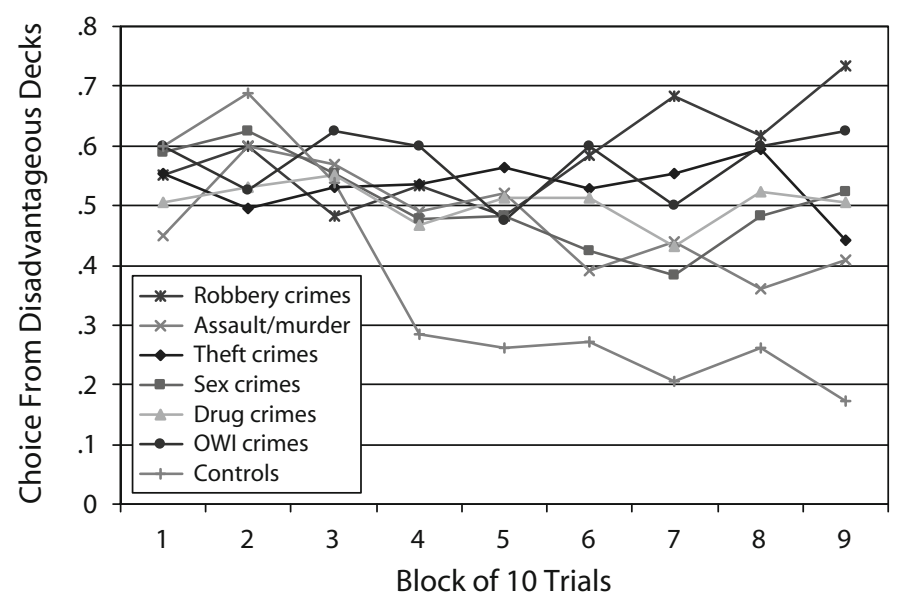

Figure 1. Average choices from disadvantageous alternatives in the Iowa gambling task by different criminal offenders and control participants.

task experience (10 trial blocks) as a repeated measure, and IQ as a covariate showed a significant group difference $\left[F(6,91)=4.90, M S_{\mathrm{e}}=0.15, p<.01\right]$, as well as a group $\times$ task experience interaction $[F(6,91)=8.90$, $\left.M S_{\mathrm{e}}=0.07, p<.01\right]$, but no main effect of experience. ${ }^{1}$ A post hoc Duncan test shows that one group was different from all of the others: The controls made fewer disadvantageous choices than did the prisoners $(p<.05)$.

To examine the distinctive psychological processes affecting choice behavior, the prisoners' choices on the IGT were analyzed using the EV model. The fit of the EV model, as compared with that of the baseline models, is described in Table 3. Comparisons were made using paired $t$ tests (with Bonferroni correction, $\alpha=.05 / 6=$ .008 for the criminal subpopulations). The average fit of the EV model was generally better than that of all three baseline models. It was significantly better than the fit of the two Softmax models for both the incarcerated individuals and the control group and was significantly better than the fit of the statistical model for the control group. There were specific subgroups in which the EV was inferior to one of the baseline models. For instance, the Softmax model was slightly better for the robbery and assault criminals. However, the subpopulation differences were not significant. We considered these fit differences as justifying the use of the EV model for interpreting the participants' behavior. ${ }^{2}$ We therefore compared the values of the EV model parameters for the different subgroups. These parameters are theoretically derived estimates of three psychological components implicated in IGT decisions: (1) attention to gains over losses, (2) influence of recent over prior outcomes, and (3) choice consistency (see the Appendix).

To examine the possibility that in the criminal sample, there were subgroups that could be characterized by the model parameters, we subjected this sample to a cluster analysis on the model parameters, using the BIC criterion (Schwartz, 1978) for determining the number of clusters. The results, summarized in Figure 2, revealed two dis- tinct clusters of criminals. One cluster center denotes individuals with high attention to gains. Most OWI criminals $(75 \%)$, along with most drug $(68 \%)$, sex $(71 \%)$, and theft $(64 \%)$ criminals, fall into this cluster. The second cluster center denotes individuals with elevated weighting of recent, as compared with past, outcomes (high recency) and with low choice consistency. More individuals who conducted violent crimes, including robbery criminals (67\%) and assault/murder criminals (50\%), fall into this second cluster. Therefore, despite the similar tendency to pick more cards from the disadvantageous decks, the different prisoner groups tended to show varied patterns in the underlying cognitive processes associated with this choice behavior (however, note that the results for the robbery and assault/murder criminals should be interpreted with caution in light of their small sample size).

In addition to studying clusters determined by the estimated model parameters, we also studied the differences between existing criminal subpopulations. In Figure 3, the results for the different criminal subpopulations are compared with previous results with brain-lesioned patients and chronic cocaine abusers on the basis of their respective EV

Table 3

Model Fits: Average Differences in the Bayesian Information Criterion (BIC) Between the Expectancy Valence (EV)

Model and Three Alternative Models: The Simple Softmax Model, the Softmax-Gains/Losses Model, and the Statistical Baseline Model

\begin{tabular}{lccc}
\hline \multicolumn{1}{c}{ Group } & Softmax & $\begin{array}{c}\text { Softmax- } \\
\text { Gains/Losses }\end{array}$ & $\begin{array}{c}\text { Statistical } \\
\text { Model }\end{array}$ \\
\hline All Criminals (81) & $7.3^{*}$ & $9.4^{*}$ & 1.5 \\
Theft criminals (22) & 4.9 & 7.3 & -0.5 \\
OWI criminals (4) & 10.7 & 13.0 & 11.3 \\
Sex criminals (17) & 7.5 & 9.2 & 0.0 \\
Drug criminals (22) & $15.9^{* *}$ & $17.4^{* *}$ & 2.8 \\
Robbery criminals (6) & -1.1 & 2.0 & $3.8^{* *}$ \\
Assault/murder criminals (10) & -3.1 & -0.7 & 0.4 \\
Controls: Healthy volunteers (18) & $21.7^{*}$ & $22.2^{*}$ & $15.0^{*}$ \\
\hline
\end{tabular}

Note-A positive BIC indicates that the EV model performs better. ${ }^{*} p<.05 .{ }^{* *} p<.008$ (with Bonferroni correction for six comparisons). 
model parameters. Each point represents the median difference between the target group and its respective control group (see Table 2). Each median difference score is located at the center of a circle, which is positioned along two dimensions. The horizontal dimension represents differences in the attention to gains relative to losses, and the vertical dimension represents differences in the influence of recent versus past outcomes. The standard errors of the difference are denoted by a cross beginning at the center of each circle. The radius of each circle represents differences in the choice consistency parameter (i.e., small bubbles denote populations with low choice consistency).

The figure indicates that drug, sex, OWI, and theft criminals were characterized by high attention to gains, being similar to the chronic cocaine abusers. The differences from controls on this parameter were statistically significant for drug criminals (Mann-Whitney $Z=2.05, p<.05$ ) and for the sex offenders (Mann-Whitney $Z=2.63, p<.01$ ). ${ }^{3}$

The median split of the assault/murder criminals was most similar to the orbitofrontal-lesioned patients, being characterized by relatively low choice consistency and by focusing on the most recent trials. Robbery criminals shared the elevated influence of recent over past outcomes and low choice consistency but were distinguished by an even more extreme focus on recent outcomes and by lower attention to gains versus losses. A separate comparison of the robbery and the assault/murder criminals with controls did not reveal significant differences, but this could have been due to the small sample sizes in these groups. If we cautiously treat these two subpopulations as a group, then as compared with controls, it is characterized by lower choice consistency (Mann-Whitney $Z=1.93, p=.05$ ) as well as by no difference in the attention to gains (Mann-Whitney $Z=-0.02$, n.s.). The average ranked recency for robbery and assault/murder criminals was not significantly different from that for controls, but the former included more decision makers with extremely high recency $(\phi>0.2)$ - that is, $65 \%$ high-recency individuals, as compared with $39 \%$ in the control groups (binomial test, $p<.05$ ). Note, however, that because the combination of the robbery and assault/ murder criminals into one group was not a priori, their significant test results should be interpreted with caution.

\section{DISCUSSION}

The present findings shed light on the similarities and differences among criminals incarcerated for different offenses. Our results provide support for the similarity hypothesis (Gottfredson \& Hirschi, 1990), in that criminals, in general, made poor decisions characterized by a failure to learn from repeated mistakes (see Figure 1). However, the results also demonstrate that different types of criminals exhibit distinct patterns of psychological deficits that are responsible for their outwardly similar choice behavior.

Specifically, the present study indicates that drug and sex offenders, and, to some extent, OWI and theft criminals as well, behaved similarly to chronic cocaine abusers. As

\begin{tabular}{|l|c|c|c|}
\hline Cluster & Recency & Att. Gains & Consistency \\
\hline Gains + & $0.11(0.14)$ & $0.81(0.19)$ & $0.11(0.14)$ \\
\hline Recency + Consistency - & $0.81(0.31)$ & $0.31(0.42)$ & $-0.19(0.18)$ \\
\hline
\end{tabular}

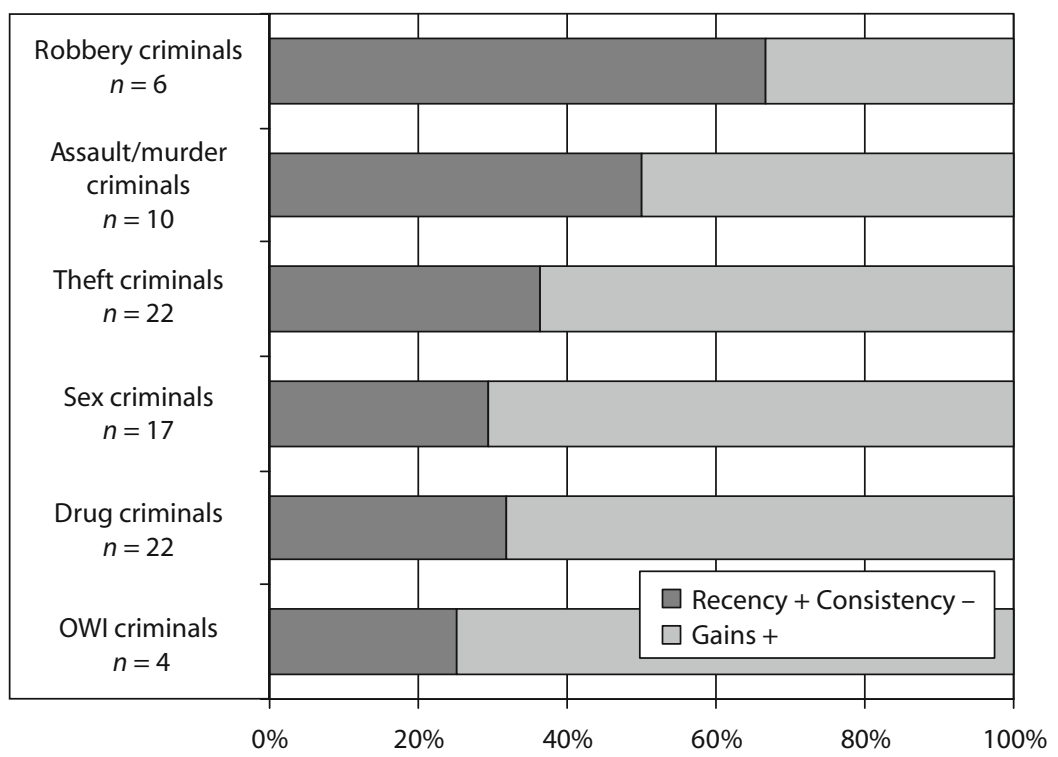

Figure 2. Results of cluster analysis based on the cognitive model parameters: Means and standard deviations (in parentheses) of the centroids of the two clusters (top) and percentage of criminals falling into each cluster (bottom). Att. gains, attention to gains. 


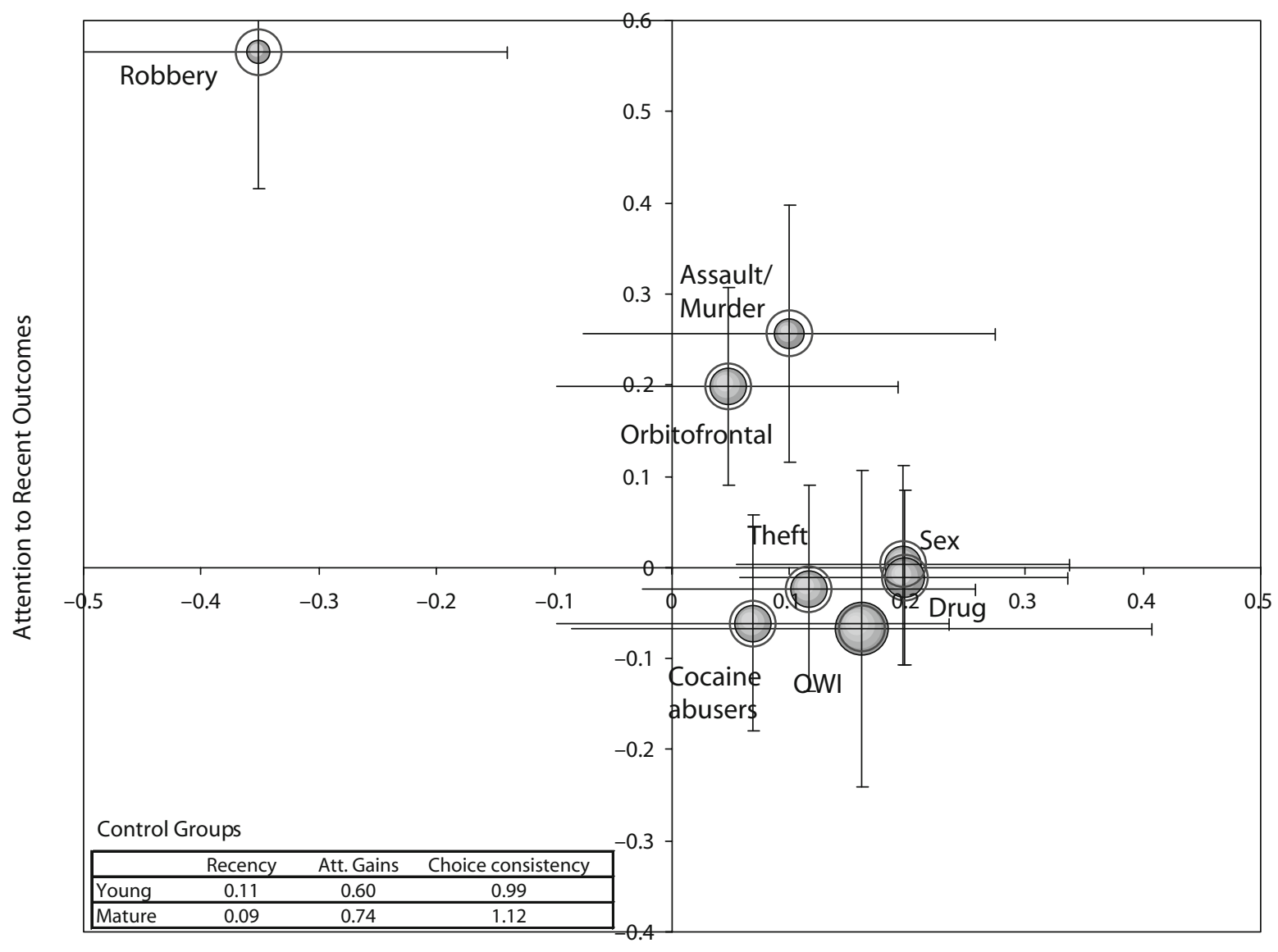

Attention to Gains

Figure 3. Mapping of studied populations according to differences in attention to loss/gain parameter and recency parameter, as compared with controls (medians and standard errors of the difference). The volume of the bubbles is proportional to the difference in the choice consistency parameter. The ring around the bubbles denotes the zero difference boundary (bubbles smaller than the ring denote populations with low choice consistency). The table summarizes the parameters of the two control groups.

compared with controls, these groups weighted gains more than losses, whereas they did not differ from controls on the influence of recent versus past outcomes or on choice consistency. Cocaine abuse has been linked to a reduction in the level of the neurotransmitter dopamine, which has a central role in reward learning: Using cocaine results in an increase in exhilaration to immediate gains (McGregor \& Roberts, 1993). It is therefore not surprising that forms of crimes characterized by addiction (e.g., drug and sex crimes) are associated with a similar tendency to prefer alternatives that produce high gains and discount their potential losses.

In contrast, the violent and murder-related criminals tended to be characterized by low choice consistency and by giving more weight to recent versus past outcomes (one should note that this was found to be statistically significant only when the two groups were combined). This tendency would imply that these decision makers have difficulties in integrating past and present information and in planning ahead. This implication is consistent with two studies showing that restlessness and problems in concentration predicted future violent offenses in teen- agers (Farrington, Loeber, \& van Kammen, 1990; Klinteberg, Andersson, Magnusson, \& Stattin, 1993) and with findings that extremely violent offenders have impaired memory for emotional events (Dollan \& Fullam, 2005).

The similarity between the neurocognitive profile of assault/murder criminals and patients with orbitofrontal damage is consistent with Raine's (2002) view that the key neural abnormalities in violent criminals lie in a prefrontal cortex deficit. For example, Raine et al. (2000) found reduced prefrontal glucose metabolism in 41 murderers, as compared with 41 matched normal controls. The robbery criminals overlapped with the orbitofrontal patients in that they also had an increasingly erratic performance and an elevated influence of recent versus past outcomes. However, their high recency was more extreme than that of the assault/murder criminals. Given the premeditated and violent nature of robbery offenses, the similarity of robbers to assault/murder criminals is not surprising.

The results of this study demonstrate the utility of cognitive models in providing a microanalysis of overall task performance patterns. The examination of repeated (or 
experience-based) choice behavior is extremely important in studies of clinical populations (see Bechara et al., 1994; Leland \& Grafman, 2005). However, usually, choice proportions that are averaged across all trials are used, even though these tasks provide numerous observations for each participant. The results of the present study show that trial-to-trial choices and outcomes can be used to analyze the elaborate cognitive processes shaping the individual's choice behavior.

\section{AUTHOR NOTE}

This research was supported in part by Grant 501-113801 from the National Science Foundation and by Grants PO1 NS19632 (NINDS) and RO1 DA11779 (NIDA) from the National Institutes of Health. We thank Steve Grant for sharing results on cocaine abusers in a previous work cited here. Correspondence concerning this article should be addressed to J. S. Paulsen, Department of Neurology, University of Iowa, 200 Hawkins Drive \#2880 JPP, Iowa City, IA 52242-1057 (e-mail: jane-paulsen@uiowa.edu).

\section{REFERENCES}

ARMSTRONG, T. (2005). Evaluating the competing assumptions of Gottfredson and Hirschi's (1990) A general theory of crime and psychological explanations of aggression. Western Criminology Review, 6 , 12-21.

Bechara, A., Damasio, A. R., Damasio, H., \& Anderson, S. (1994). Insensitivity to future consequences following damage to human prefrontal cortex. Cognition, 50, 7-15.

Bechara, A., Damasio, H., \& Damasio, A. R. (2000). Emotion, decision making and the orbitofrontal cortex. Cerebral Cortex, 10, 295-307.

Bechara, A., Damasio, H., Damasio, A. R., \& Lee, G. P. (1999). Different contributions of the human amygdala and ventromedial prefrontal cortex to decision-making. Journal of Neuroscience, 19, 5473-5481.

Busemeyer, J. R., \& Stout, J. C. (2002). A contribution of cognitive decision models to clinical assessment: Decomposing performance on the Bechara gambling task. Psychological Assessment, 14, 253-262.

Caroselli, J. S., Hiscock, M., Scheibel, R., \& Ingram, F. (2006). The simulated gambling paradigm applied to young adults: An examination of university students' performance. Applied Neuropsychology, 13, 203-212.

Daw, N. D., O'Doherty, J. P., Dayan, P., Seymour, B., \& Dolan, R. J. (2006). Cortical substrates for exploratory decisions in humans. $\mathrm{Na}$ ture, 441, 876-879.

Dollan, M., \& Fullam, R. (2005). Memory for emotional events in violent offenders with antisocial personality disorder. Personality \& Individual Differences, 38, 1657-1667.

Farrington, D. P., Loeber, R., \& VAn Kammen, W. B. (1990). Longterm criminal outcomes of hyperactivity-impulsivity-attention deficit and conduct problems in childhood. In L. N. Robins \& M. Rutter (Eds.), Straight and devious pathways from childhood to adulthood (pp. 62-81). Cambridge, MA: Cambridge University Press.

GotTfredson, M. R., \& Hirschi, T. A. (1990). A general theory of crime. Stanford, CA: Stanford University Press.

Hope, T. L., \& DAmphouse, K. R. (2002). Applying self-control theory to gang membership in a non-urban setting. Journal of Gang Research, 9, 41-61.
Keane, C., Maxim, P. S., \& Teevan, J. J. (1993). Drinking and driving, self-control, and gender: Testing a general theory of crime. Journal of Research in Crime \& Delinquency, 30, 30-46.

KellogG, C. E., \& Morton, N. W. (1974). Revised Beta ExaminationSecond edition manual (Beta II). New York: Psychological Corp.

Klinteberg, B. A., Andersson, T., Magnusson, D., \& Stattin, H. (1993). Hyperactive behavior in childhood as related to subsequent alcohol problems and violent offending: A longitudinal study of male subjects. Personality \& Individual Differences, 15, 381-388.

Krueger, R. F., Hicks, B. M., \& McGue, M. (2001). Altruism and antisocial behavior: Independent tendencies, unique personality correlates, distinct etiologies. Psychological Science, 12, 397-402.

Leland, J. W., \& Grafman, J. (2005). Experimental tests of the somatic marker hypothesis. Games \& Economic Behavior, 52, 386-409.

Lombroso, C. (2005). Criminal man. Durham, NC: Duke University Press. (Original work published 1911)

McGregor, A., \& RoberTs, D. C. S. (1993). Dopaminergic antagonism within the nucleus accumbens or the amygdala produces differential effects on intravenous cocaine self-administration under fixed and progressive ratio schedules of reinforcement. Brain Research, 624, 245-252.

RaINE, A. (2002). Annotation: The role of prefrontal deficits, low autonomic arousal, and early health factors in the development of antisocial and aggressive behavior in children. Journal of Child Psychology \& Psychiatry, 43, 417-434.

Raine, A., Lencz, T., Bihrle, S., LaCasse, L., \& Colletti, P. (2000). Reduced prefrontal gray matter volume and reduced autonomic activity in antisocial personality disorder. Archives of General Psychiatry, 57, 119-127.

Schwartz, G. (1978). Estimating the dimension of a model. Annals of Statistics, 5, 461-464.

Stout, J. C., Busemeyer, J. R., Lin, A., GRAnt, S. R. J., \& Bonson, K. R. (2004). Cognitive modeling analysis of decision-making processes in cocaine abusers. Psychonomic Bulletin \& Review, 11, 742-747.

Volkow, N. D., Wang, G.-J., Fischman, M. W., Foltin, R. W., Fowler, J. S., AbumRad, N. N., ET AL. (1997). Relationship between subjective effects of cocaine and dopamine transporter occupancy. Nature, 386, 827-830.

YeCHIAM, E., \& BUSEMEYER, J. R. (in press). Evaluating parameter consistency in learning models. Games \& Economic Behavior.

Yechiam, E., Busemeyer, J. R., Stout, J. C., \& Bechara, A. (2005). Using cognitive models to map relations between neuropsychological disorders and human decision making deficits. Psychological Science, 16, 973-978.

\section{NOTES}

1. Beta IQ was a significant covariate $\left[F(1,91)=4.90, M S_{\mathrm{e}}=0.15\right.$, $p<.01]$ as was the interaction between IQ and task experience $[F(1,91)=$ $\left.4.14, M S_{\mathrm{e}}=0.07, p<.05\right]$, with low-IQ criminals making fewer advantageous selections in repeated choices.

2 . Note that to fully evaluate the different models, more than one task should be administered, since parameter generalizability should also be considered. Studies in which the EV model has been examined using multiple tasks have shown that individual differences in the attention to gains and recency parameters are consistent in different tasks (see Yechiam \& Busemeyer, in press).

3 . These two significant differences are replicated when using a parametric analysis and covarying for IQ. 


\section{APPENDIX}

The EV model is composed of three basic components.

1. Attention to wins versus losses is measured by the attention weight parameter. The evaluation of the gains and losses experienced after making a choice is called a valence. The valence is denoted $u(t)$ and is calculated as a weighted average of gains and losses from the chosen option in trial $t$ :

$$
u(t)=w \cdot \operatorname{win}(t)-(1-w) \cdot \operatorname{loss}(t),
$$

where $\operatorname{win}(t)$ and $\operatorname{loss}(t)$ are the amounts of money won or lost on trial $t$ and $w$ is the attention weight parameter indicating the subjective weight to gains versus losses $(0 \leq w \leq 1)$.

2. Influence of recent versus past outcomes is measured by the recency parameter. The valences produced by a deck $j$ are summarized by an accumulated subjective value for each deck, called an expectancy and denoted $E_{j}(t)$. A delta learning rule is used for updating the expectancy after each choice:

$$
E_{j}(t)=E_{j}(t-1)+\phi \cdot\left[u(t)-E_{j}(t-1)\right],
$$

where $j$ is the selected deck. The recency parameter, $\phi$, describes the degree to which expectations about deck consequences reflect the influence of the most recent outcomes or more distant past experience $(0 \leq \phi \leq 1)$.

3. Sensitivity of responses to expectancies is measured by the choice consistency parameter. The probability of choosing a deck is a strength ratio of the expectancy of that deck relative to all decks (using Luce's rule):

$$
\operatorname{Pr}\left[G_{j}(t+1)\right]=\frac{e^{\theta(t) \cdot E_{j}(t)}}{\sum_{j} e^{\theta(t) \cdot E_{j}(t)}},
$$

where $\operatorname{Pr}\left[G_{j}(t)\right]$ is defined as the probability that deck $j$ will be selected on trial $t$ by the model. The term $\theta(t)$ controls the consistency of the choice probabilities and the expectancies, where $\theta(t)=(t / 10)^{c}$ and $c$ is the choice consistency parameter.

The EV model was compared with two alternative learning models.

1. The first is the Softmax model (e.g., Daw et al., 2006), which assumes that the probability of choosing an alternative is a function of the difference between the experienced expected values (i.e., the mean payoff values by trial $t$ ) of the alternatives. For conciseness, we employed a model using Equation A3, in which the experienced expected values replace the expectancies. This model has only a single free parameter ( $c$, as above).

2. A more sophisticated Softmax model (called here Softmax-gains/losses) is one in which individuals give different weights to gains and losses (as in Equation A1). Thus, this model has the same parameters, $w$ and $c$, as in the EV model but no recency parameter.

The two Softmax models therefore provide a test for the need to add the recency parameter, instead of basing the expectancies on expected values or expected valences (in the simple and sophisticated models, respectively). 